\title{
Modelo de Cercignani-Lampis para a interação gás-superfície aplicado ao cálculo da força radiométrica em uma esfera imersa em um gás rarefeito.
}

\author{
Denize Kalempa, ${ }^{1}$ \\ Paulo, Lorena, SP. \\ Felix Sharipov ${ }^{2}$ \\ Departamento de Física,Universidade Federal do Paraná, Curitiba, PR.
}

Departamento de Ciências Básicas e Ambientais, Escola de Engenharia de Lorena, Universidade de São

Resumo. No presente trabalho, a força radiométrica que atua em uma esfera com temperatura não-uniforme é calculada com base na solução numérica de um modelo cinético para a equação de Boltzmann linearizada e no modelo de Cercignani-Lampis para a interação gás-superfície. A força que atua na esfera, assim como os campos de escoamento induzidos pela distribuição de temperatura na superfície esférica, são obtidos em um amplo intervalo de número de Knudsen, definido como a razão entre o livre caminho médio molecular e o raio da esfera, tal que os regimes de moléculas livres, transição e contínuo são considerados. Além disso, visando analisar a influência da lei de interação gás-superfície na solução do problema, vários valores de coeficientes de acomodação são considerados.

Palavras-chave. Força radiométrica, equação de Boltzmann, aerossóis, gás rarefeito, interação gás-superfície.

\section{Introdução}

Atualmente, o crescente interesse por estudos relacionados à força radiométrica em sistemas gasosos se deve ao desenvolvimento de micro e nano tecnologias tais como sensores de gás $[5,8]$. No contexto de teoria cinética dos gases, um escoamento gasoso pode ser induzido por um campo de temperatura mesmo na ausência de forças externas, e.g. o famoso rastejo térmico [7,15], do inglês thermal creep. Porém, o escoamento de um gás também pode resultar em um efeito térmico, e.g. a polarização de uma esfera imersa em um gás rarefeito em movimento com velocidade uniforme [1]. O efeito de polarização térmica da esfera leva ao aparecimento de uma força na esfera, denominada força radiométrica devido aos famosos experimentos de Crookes [4].

No presente trabalho a força radiométrica que atua em uma esfera termicamente polarizada, com tamanho e condutividade térmica arbitrários, é calculada numericamente com base no modelo proposto por Shakhov [12] para a equação linearizada de Boltzmann. Visando analisar a influência da interação gás-superfície na solução do problema, o modelo proposto por Cercignani e Lampis [3] é utilizado na condição de contorno. Geralmente, o modelo proposto por Maxwell [9] é utilizado em problemas envolvendo escoamento de gases rarefeitos. De acordo com o modelo de Maxwell, uma fração $\alpha$ das partículas gasosas é refletida difusamente e o restante $1-\alpha$ especularmente.

\footnotetext{
${ }^{1}$ kalempa@usp.br

2 sharipov@fisica.ufpr.br
} 
Porém, apesar da sua simplicidade matemática, esse modelo de interação gás-superfície pode fornecer resultados em discordância com dados experimentais. No modelo de Cercignani-Lampis, dois coeficientes de acomodação são introduzidos, um coeficiente de acomodação de momentum tangencial e outro coeficiente de acomodação de energia. Portanto, apesar da sua complexidade matemática, o modelo de Cercignani-Lampis fornece uma melhor descrição física do processo de interação gás-superfície já que dois coeficientes de acomodação são introduzidos.

Os campos de escoamento do gás ao redor da esfera, i.e. desvios de densidade e temperatura, velocidade hidrodinâmica e fluxo de calor, induzidos pela distribuição de temperatura não uniforme da esfera também são calculados. De acordo com a literatura, e.g. Refs. [16,18], uma peculiaridade de escoamentos rarefeitos induzidos por campos de temperatura é a usência de movimento do gás no regime de moléculas livres quando a hipótese de espalhamento difuso é utilizada. Porém, no presente trabalho, mostramos que no regime de moléculas livres existe movimento do gás ao redor da esfera quando outra lei de interação gás-superfície é utilizada.

\section{Formulação do problema}

Consideramos uma esfera estacionária de raio $R_{0}$ e condutividade térmica $\lambda_{p}$ em um gás rarefeito monoatômico de condutividade térmica $\lambda_{g}$. Longe da esfera assumimos que o gás está em equilíbrio termodinâmico com densidade de número, pressão e temperatura denotados, respectivamente, por $n_{0}, p_{0}$ e $T_{0}$, e escoa com velocidade constante $U_{\infty}$ na direção $z^{\prime}$. Devido à geometria do problema, coordenadas esféricas $\left(r^{\prime}, \theta, \phi\right)$ são introduzidas no espaço físico $\mathbf{r}^{\prime}$ e $\left(v, \theta^{\prime}, \phi^{\prime}\right)$ no espaço de velocidade molecular $\mathbf{v}$. Detalhes podem ser encontrados em nosso trabalho anterior [6]. Por conveniência, introduzimos a coordenada radial e a velocidade molecular adimensionais

$$
r=\frac{r^{\prime}}{\ell_{0}}, \quad \mathbf{c}=\frac{\mathbf{v}}{v_{0}}
$$

onde $\ell_{0}=\mu_{0} v_{0} / p_{0}$ é uma distância equivalente ao livre caminho médio molecular e $v_{0}=\sqrt{2 k T_{0} / m}$ é a velocidade molecular mais provável. $k$ é a constante de Boltzmann e $m$ é a massa molecular.

O parâmetro de rarefação do gás, o qual é inversamente proporcional ao número de Knudsen, é definido aqui como

$$
\delta=\frac{R_{0}}{\ell_{0}}=r_{0}
$$

Dependendo do valor do parâmetro de rarefação, o regime de escoamento do gás é classificado em regime de moléculas livres $(\delta \ll 1)$, contínuo ou hidrodinâmico $(\delta \gg 1)$ e de transição $(\delta \sim 1)$.

Assumimos que a razão entre as condutividades térmicas da esfera e do gás, $\Lambda=\lambda_{p} / \lambda_{g}$, é arbitrária. Nessa situação, a distribuição de temperatura na superfície da esfera precisa ser considerada na condição de contorno. Essa distribuição de temperatura é obtida da solução da equação de Laplace com simetria axial e, para o problema em questão, é escrita do seguinte modo

$$
T_{p}\left(R_{0}, \theta\right)=T_{0}\left(1+\tau_{s 0} \cos \theta\right),
$$

onde $\tau_{s 0}$ é uma constante.

Duas forças termodinâmicas são introduzidas,

$$
X_{u}=\left|\frac{U_{\infty}}{v_{0}}\right| \ll 1, \quad X_{q}=\left|\tau_{s 0}\right| \ll 1,
$$

as quais são acopladas devido à polarização térmica da esfera. Entretanto, como assumimos que as suas magnitudes são muito pequenas, o problema pode ser dividido em duas partes independentes: 
(i) arrasto viscoso com temperatura da esfera uniforme e (ii) força radiométrica induzida pela distribuição não-uniforme de temperatura na superfície esférica com gás estacionário. Assim, cada problema pode ser resolvido separadamente e, posteriormente, a força total que atua na esfera é dada pela superposição das soluções que, na forma adimensional, é escrita como

$$
F_{z}=\frac{F_{z}^{\prime}}{4 \pi R_{0}^{2} p_{0}}=F_{u} X_{u}+F_{q} X_{q}
$$

onde $F_{u}$ e $F_{q}$ denotam, respectivamente, as forças de arrasto viscoso e radiométrica adimensionais. $F_{z}^{\prime}$ denota a força total, dimensional, na esfera. De fato, o problema de arrasto viscoso em uma esfera com temperatura uniforme, que corresponde à consideração de uma esfera com condutividade térmica muito maior que a condutividade térmica do gás, já foi encontrada na Ref. [6]. Portanto, nesse trabalho foco é dado ao cálculo da força radiométrica na esfera. É importante mencionar que, apesar da força radiométrica ser desprezível quando comparada com a força de arrasto viscoso, a introdução das duas forças termodinâmicas definidas em (4) permite utilizarmos a relação de reciprocidade entre os efeitos acoplados como um critério adicional para a verificação da convergência dos cálculos numéricos.

\section{Método de solução}

O problema é resolvido com base no modelo proposto por Shakhov [12] para a equação linearizada de Boltzmann [2] e no método de velocidades discretas, o qual é amplamente utilizado na área de dinâmica de gases rarefeitos e cujos detalhes podem ser encontrados na Ref. [13]. De fato, a solução do problema é similar à apresentada em nosso trabalho anterior [6], mas a condição de contorno é modificada para a consideração da distribuição de temperatura na superfície esférica devida à polarização térmica da esfera. Então, seguindo a mesma notação da Ref. [6], a equação cinética para o problema axi-simétrico em questão (simetria em $\phi$ ) é escrita na forma adimensional do seguinte modo:

$$
\hat{D} h^{(q)}=\hat{L}_{S} h^{(q)},
$$

onde o operador de transporte é dado por

$$
\hat{D} h^{(q)}=c_{r} \frac{\partial h^{(q)}}{\partial r}-\frac{c_{t}}{r} \frac{\partial h^{(q)}}{\partial \theta^{\prime}}+\frac{c_{t}}{r} \cos \phi^{\prime} \frac{\partial h^{(q)}}{\partial \theta}-\frac{c_{t}}{r} \sin \phi^{\prime} \cot \theta \frac{\partial h^{(q)}}{\partial \phi^{\prime}}
$$

e o operador de colisões é dado por

$$
\hat{L}_{S} h^{(q)}=\nu^{(q)}+\left(c^{2}-\frac{3}{2}\right) \tau^{(q)}+2 \mathbf{c} \cdot \mathbf{u}^{(q)}+\frac{4}{15}\left(c^{2}-\frac{5}{2}\right) \mathbf{c} \cdot \mathbf{q}^{(q)}-h^{(q)} .
$$

A função $h^{(q)}=h^{(q)}(r, \theta, \mathbf{c})$ é chamada de função perturbação e representa o desvio da função de distribuição de velocidades moleculares da função de Maxwell característica do estado de equilíbrio termodinâmico do sistema gasoso. No operador de colisões (8), as quantidades

$$
\begin{gathered}
\nu^{(q)}(r, \theta)=\frac{1}{\pi^{3 / 2}} \int h^{(q)}(r, \theta, \mathbf{c}) \mathrm{e}^{-c^{2}} d \mathbf{c}, \\
\tau^{(q)}(r, \theta)=\frac{2}{3 \pi^{3 / 2}} \int\left(c^{2}-\frac{3}{2}\right) h^{(q)}(r, \theta, \mathbf{c}) \mathrm{e}^{-c^{2}} d \mathbf{c},
\end{gathered}
$$

correspondem aos desvios de densidade e temperatura do gás dos valores correspondendo ao equilíbrio termodinâmico, e

$$
u_{r}^{(q)}(r, \theta)=\frac{1}{\pi^{3 / 2}} \int c_{r} h^{(q)}(r, \theta, \mathbf{c}) \mathrm{e}^{-c^{2}} d \mathbf{c},
$$




$$
\begin{gathered}
u_{\theta}^{(q)}(r, \theta)=\frac{1}{\pi^{3 / 2}} \int c_{\theta} h^{(q)}(r, \theta, \mathbf{c}) \mathrm{e}^{-c^{2}} d \mathbf{c}, \\
q_{r}^{(q)}(r, \theta)=\frac{1}{\pi^{3 / 2}} \int c_{r}\left(c^{2}-\frac{5}{2}\right) h^{(q)}(r, \theta, \mathbf{c}) \mathrm{e}^{-c^{2}} d \mathbf{c}, \\
q_{\theta}^{(q)}(r, \theta)=\frac{1}{\pi^{3 / 2}} \int c_{\theta}\left(c^{2}-\frac{5}{2}\right) h^{(q)}(r, \theta, \mathbf{c}) \mathrm{e}^{-c^{2}} d \mathbf{c},
\end{gathered}
$$

correspondem às componentes radial e polar dos vetores velocidade hidrodinâmica $\mathbf{u}$ e fluxo de calor q. Ademais, $c_{r}=c \cos \theta^{\prime}$ e $c_{t}=c \sin \theta^{\prime}$ denotam as componentes radial e tangencial do vetor velocidade molecular adimensional, definido em (1), e $d \mathbf{c}=c^{2} \sin \theta^{\prime} d c d \theta^{\prime} d \phi^{\prime}$.

A força radiométrica, adimensional, que atua na esfera é calculada como

$$
F_{q}=-\frac{1}{2 \pi^{5 / 2}} \int_{\Sigma_{w}} \mathrm{~d} \Sigma_{w} \int c_{r} c_{z} \mathrm{e}^{-c^{2}} h^{(q)}\left(r_{0}, \theta, \mathbf{c}\right) d \mathbf{c}
$$

onde $\mathrm{d} \Sigma_{w}=\sin \theta \mathrm{d} \theta \mathrm{d} \phi$ denota um elemento de área infinitesimal na superfície da esfera.

De acordo com as Refs. $[2,13]$, a condição de contorno, em $r=r_{0}$, utilizada para resolver a equação integro-diferencial (6) é a seguinte:

$$
h^{+(q)}=\hat{A} h^{-(q)}+h_{w}^{(q)}-\hat{A} h_{w}^{(q)}, \quad h_{w}^{(q)}=\left(c^{2}-\frac{3}{2}\right) \frac{z_{0}}{\delta} \cos \theta
$$

onde o sinal " + " indica partículas gasosas (átomos ou moléculas) refletidas e o sinal " partículas gasosas incidentes, $c_{z}=c_{r} \cos \theta-c_{\theta} \sin \theta$ e $z_{0}=r_{0} \cos \theta$.

Para o problema em questão, o operador de espalhamento $\hat{A}$ é decomposto como

$$
\hat{A} h^{(q)}=\hat{A}_{r} \hat{A}_{\theta} \hat{A}_{\phi} h^{(q)}
$$

onde

$$
\begin{aligned}
& \hat{A}_{r} \xi=-\frac{1}{c_{r}} \int_{c_{r}^{\prime}<0} c_{r}^{\prime} \exp \left(c_{r}^{2}-c_{r}^{\prime 2}\right) R_{r}\left(c_{r} \rightarrow c_{r}^{\prime}\right) \xi\left(c_{r}^{\prime}\right) \mathrm{d} c_{r}^{\prime}, \\
& \hat{A}_{i} \xi=\int_{-\infty}^{\infty} \exp \left(c_{i}^{2}-c_{i}^{\prime 2}\right) R_{i}\left(c_{i} \rightarrow c_{i}^{\prime}\right) \xi\left(c_{i}^{\prime}\right) \mathrm{d} c_{i}^{\prime}, \quad i=\theta, \phi,
\end{aligned}
$$

para uma função arbitrária $\xi$ da velocidade molecular c. De acordo com o modelo de Cercignani e Lampis [3],

$$
\begin{gathered}
R_{r}\left(c_{r} \rightarrow c_{r}^{\prime}\right)=\frac{2 c_{r}}{\alpha_{n}} \exp \left[-\frac{c_{r}^{2}+\left(1-\alpha_{n}\right) c_{r}^{\prime 2}}{\alpha_{n}}\right] I_{0}\left(\frac{2 \sqrt{1-\alpha_{n}}}{\alpha_{n}} c_{r} c_{r}^{\prime}\right), \\
R_{i}\left(c_{i} \rightarrow c_{i}^{\prime}\right)=\frac{1}{\sqrt{\pi \alpha_{t}\left(2-\alpha_{t}\right)}} \exp \left\{-\frac{\left[c_{i}-\left(1-\alpha_{t}\right) c_{i}^{\prime}\right]^{2}}{\alpha_{t}\left(2-\alpha_{t}\right)}\right\}, \quad i=\theta, \phi,
\end{gathered}
$$

onde $I_{0}$ denota a função modificada de Bessel de primeira espécie e ordem zero. $\alpha_{t}$ denota o coeficiente de acomodação de momentum tangencial e $\alpha_{n}$ denota o coeficiente de acomodação de energia, os quais podem variar nos intervalos $0 \leq \alpha_{t} \leq 2$ e $0 \leq \alpha_{n} \leq 1$. O caso $\alpha_{t}=1$ and $\alpha_{n}=1$ corresponde ao espalhamento difuso ou completa acomodação na superfície. Já o caso $\alpha_{t}=0$ e $\alpha_{n}=0$ corresponde à reflexão especular na superfície.

Além da condição de contorno, a solução da equação (6) deve satisfazer a seguinte condição longe da esfera

$$
h_{\infty}^{(q)}=\lim _{r \rightarrow \infty} h^{(q)}(r, \theta, \mathbf{c})=0,
$$




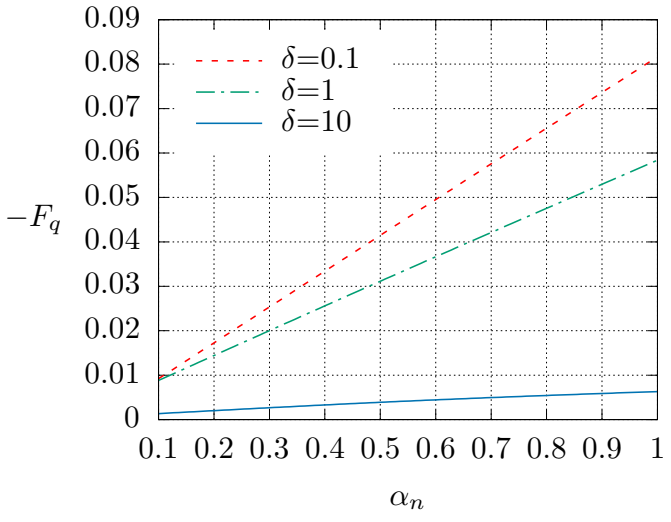

(a) $\alpha_{t}=1$

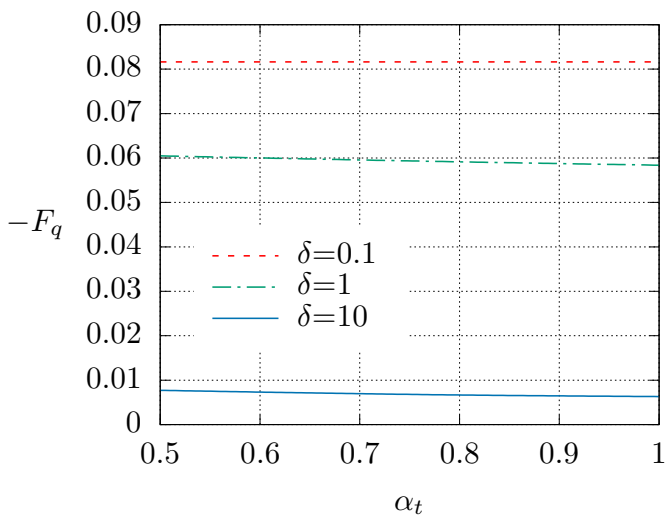

(b) $\alpha_{n}=1$

Figura 1: Influência dos coeficientes de acomodação na força radiométrica que atua na esfera.

a qual implica em equilíbrio termodinâmico.

Com o objetivo de eliminar a dependência da solução nas variáveis $\theta$ e $\phi^{\prime}$, a solução de similaridade proposta na Ref. [17] é utilizada. Assim, a função perturbação é representada do seguinte modo

$$
h^{(q)}(r, \theta, \mathbf{c})=h_{c}^{(q)}\left(r, c, \theta^{\prime}\right) \cos \theta+h_{s}^{(q)}\left(r, c, \theta^{\prime}\right) c_{\theta} \sin \theta .
$$

Consequentemente, a substituição de (23) na equação (6) fornece duas equações acopladas para as funções $h_{c}^{(q)}$ e $h_{s}^{(q)}$ com condições de contorno, e também em $r \rightarrow \infty$, obtidas de (16) e (22). Os momentos dados em (9)-(14), os quais correspondem ao campos de escoamento do gás ao redor da esfera induzidos pela distribuição de temperatura na superfície, também são reescritos em termos das funções $h_{c}^{(q)}$ e $h_{s}^{(q)}$.

O sistema de equações obtido foi resolvido numericamente utilizando o método de velocidades discretas. Os resultados obtidos para a força na esfera são apresentados a seguir para alguns valores de parâmetro de rarefação e coeficientes de acomodação.

\section{Resultados}

A Figura 1 apresenta a força radiométrica na esfera para parâmetro de rarefação $\delta=0.1,1$ e 10 . Na Figura 1(a), o coeficiente de acomodação de momentum tangencial é fixo, $\alpha_{t}=1$, e o coeficiente de acomodação de energia, $\alpha_{n}$, varia de 0.1 até 1 . Na Figura 1(b), o coeficiente de acomodação de energia é fixo, $\alpha_{n}=1$, e o coeficiente de acomodação de momentum tangencial, $\alpha_{t}$, varia de 0.5 até 1. Assim, as figuras permitem observarmos como a força varia do valor correspondendo ao caso de espalhamento difuso, para o qual $\alpha_{n}=1$ e $\alpha_{t}=1$.

Atualmente, os valores dos coeficientes de acomodação introduzidos no modelo de Cercignani e Lampis podem ser encontrados na literatura para vários gases e superfícies, e.g. Refs. [10,11, 14,19]. Em superfícies metálicas, por exemplo, o coeficiente de acomodação de energia, $\alpha_{n}$, é aproximadamente igual a 0.1 para gás hélio e está entre 0.5 e 0.95 para gás argônio, à temperatura ambiente. Nas mesmas condições, o coeficiente de acomodação de momentum tangencial, $\alpha_{t}$, varia de 0.5 a 0.95 para ambos os gases.

De acordo com a Figura 1, a dependência da força radiométrica no coeficiente de acomodação de energia é muito maior que a dependência no coeficiente de acomodação de momentum tangencial. Portanto, dependendo do gás e da superfície, a hipótese de espalhamento difuso não é adequada 


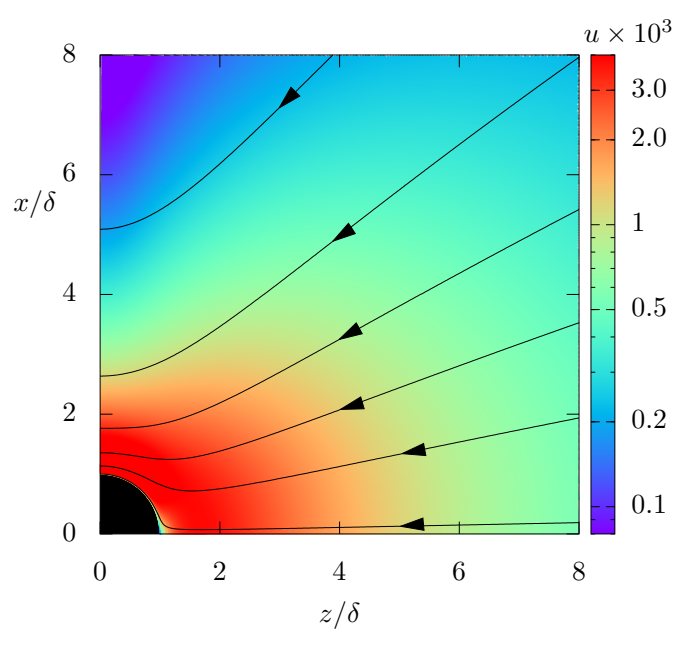

(a) $\alpha_{n}=0.1$

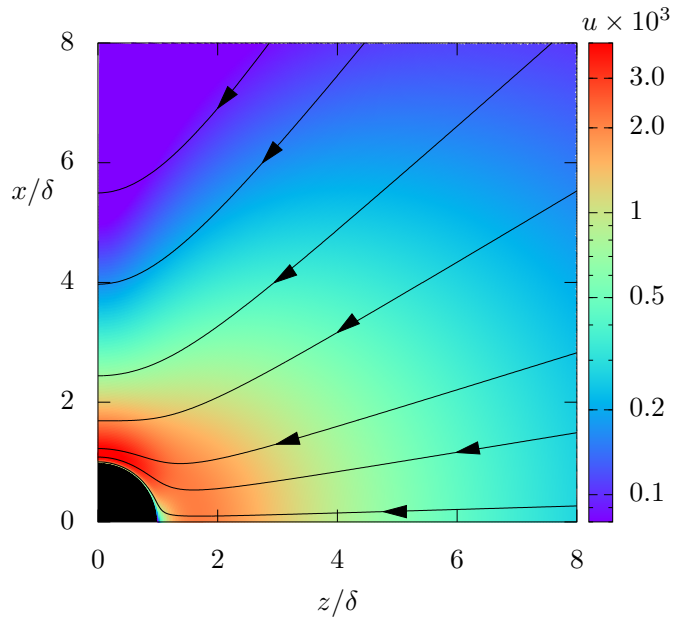

(b) $\alpha_{n}=0.5$

Figura 2: Mapa da velocidade hidrodinâmica e linhas de corrente, $\delta=0.01$ e $\alpha_{t}=1$.

para descrever a interação gás-superfície e seu uso pode fornecer resultados que não concordam com dados experimentais. Além disso, no regime de moléculas livres, a ausência de movimento do gás ao redor da esfera é prevista quando a hipótese de espalhamento difuso é usada, e.g. Ref. [18]. Porém, no presente trabalho, mostramos que para outros valores de coeficientes de acomodação há movimento do gás ao redor da esfera no regime de moléculas livres. Para visualização, na Figura 2 apresentamos o campo de velocidade do gás para $\delta=0.01, \alpha_{t}=1$ e $\alpha_{n}=0.1$ e 0.5 . Note que, de acordo com (1) e (2), $x / \delta=x^{\prime} / R_{0}$ e $z / \delta=z^{\prime} / R_{0}$, ou seja, na figura as coordenadas $x^{\prime}$ e $z^{\prime}$ são adimensionalizadas usando o raio $R_{0}$ da esfera. A figura mostra que o gás escoa na direção $-z$ e, na notação do trabalho, isso significa que o escoamento é na direção do pólo frio para o pólo quente da esfera.

A Figura 1 também mostra que a magnitude da força radiométrica tende a aumentar à medida que o parâmetro de rarefação do gás diminui, ou seja, a magnitude da força é maior no regime de moléculas livres para quaisquer valores de coeficientes de acomodação. Isso se deve ao fato de que, no regime de moléculas livres as colisões intermoleculares são desprezíveis e o único mecanismo responsável pela força na esfera é o chamado mecanismo de Knudsen, segundo o qual a força se deve à transferência de momentum das moléculas gasosas para a superfície sem influência de fenômenos inerentes às colisões intermoleculares. À medida que o parâmetro de rarefação aumenta e o gás tende aos regimes de transição e contínuo, as colisões moleculares levam a outros mecanismos que resultam em forças menores na esfera, e.g. o rastejo térmico que aparece no regime contínuo.

\section{Referências}

[1] Bakanov S. P. and Vysotskij V. Thermal polarization of bodies in the rarefied gas flow and thermophoresis of aerosols. J. Aerosol Sci., 25 SI:407-408, 1994.

[2] Cercignani C. The Boltzmann Equation and its Application. Springer-Verlag, New York, 1988.

[3] Cercignani C. and Lampis M. Kinetic model for gas-surface interaction. Transp. Theory and Stat. Phys., 1:101-114, 1971. 
[4] Crookes W. On attraction and repulsion resulting from radiation. Phil. Trans. R. Soc., 164:501-527, 1874.

[5] Hassanvand A., Gerdroodbary M. B., Moradi R. and Amini Y. Application of knudsen thermal force for detection of inert gases. Results in Physics, 9:351-358, 2018.

[6] Kalempa D. and Sharipov F. Drag and thermophoresis on a sphere in a rarefied gas based on the cercignani-lampis scattering model of gas-surface interaction. J. Fluid Mech., 900:A37, 2020.

[7] Kennard E. H. Kinetic Theory of Gases. McGraw-Hill Book Company, Inc., New York, 1938.

[8] Mahyari A., Gerdroodbary M. B., Mosavat M. and Ganji D. D. Detection of ammonia gas by knudsen thermal force in micro gas actuator. Case Studies in Thermal Engineering, 12:276-284, 2018.

[9] Maxwell J. C. On stress in rarefied gases arising from inequalities of temperature. Phil Trans. R. Soc. Lond., 170:231-256, 1879.

[10] Sazhin O. V., Borisov S. F. and Sharipov F. Accommodation coefficient of tangential momentum on atomically clean and contaminated surfaces. J. Vac. Sci. Technol. A, 19(5):2499-2503, 2001. Erratum: 20 (3), 957 (2002).

[11] Semyonov Y. G., Borisov S. F. and Suetin P. E. Investigation of heat transfer in rarefied gases over a wide range of Knudsen numbers. Int. J. Heat Mass Transfer, 27(10):1789-1799, 1984.

[12] Shakhov E. M. Generalization of the Krook kinetic relaxation equation. Fluid Dynamics, $3(5): 95-96,1968$.

[13] Sharipov F. Rarefied Gas Dynamics. Fundamentals for Research and Practice. Wiley-VCH, Berlin, 2016.

[14] Sharipov F. and Moldover M. Energy accommodation coefficient extracted from acoustic resonator experiments. J. Vac. Sci. Technol. A, 34(6):061604, 2016.

[15] Sone Y. Thermal creep in rarefied gas. J. Phys. Soc. Jpn, 21:1836-1837, 1966.

[16] Sone Y. Highly rarefied gas around a group of bodies with various temperature distributions. I - Small temperature variation. Journal de Mecanique Theorique et Appliquee, 3(2):315-328, January 1984.

[17] Sone Y. and Aoki K. A similarity solution of the linearized Boltzmann equation with application to thermophoresis of a spherical particle. Journal de Mecanique Theorique et Appliquee, 2(1):3-12, 1983.

[18] Takata S. and Sone Y. Flow induced around a sphere with a non-uniform surface temperature in a rarefied gas, with application to the drag and thermal force problem of a spherical particle with an arbitrary thermal conductivity. Eur. J. Mech. B/Fluids, 14(4):487-518, 1995.

[19] Trott W. M., Castaneda J. N., Torczynski J. R., Gallis M. A. and Rader D. J. An experimental assembly for precise measurement of thermal accommodation coefficients. Rev. Sci. Instrum., 82(3):035120, 2011. 